\title{
INDEX OF VOLUME XXXVIII
}

Adkisson, V. W. Note on the Law of Biquadratic Reciprocity, 529.

Albert, A. A. A Construction of Non-Cyclic Normal Division Algebras, 449. - A Note on Normal Division Algebras of Order Sixteen, 703.

BAsoco, M. A. On the Trigonometric Developments of Certain Doubly Periodic Functions of the Second Kind, 560.

Bateman, H. See Reviews, under Levi-Civita.

Beetle, R. D., and Wilder, C. E. John Wesley Young-In Memoriam, 603.

BeLl, E. T. On the Degree of Generality of a Class of Arithmetical Identities, 263.

- Quadratic Partitions, II, 551.

- Quadratic Partitions, III, 569.

- Quadratic Partitions, IV, 697.

- See Reviews, under Dedekind, Fulco.

Bennett, W. R. Note on Relations between Elliptic Integrals and Schlömilch Series, 843.

Bernstein, B. A. On Proposition *4.78 of Principia Mathematica, 388.

- Relation of Whitehead and Russell's Theory of Deduction to the Boolean Logic of Propositions, 589.

On Unit-Zero Boolean Representations of Operations and Relations, 707. See Reviews, under Ramsey.

Bernstein, B. A., and Debely, N. A Practical Method for the Modular Representation of Finite Operations and Relations, 110.

Birкhofr, G. D. Probability and Physical Systems, 361.

Blaschke, W. Results and Problems about $n$-Webs of Curves in a Plane, 828.

BuIss, G. A. The Calculus of Variations and the Quantum Theory, 201.

Bliss, G. A., and Schoenberg, I. J. On the Derivation of Necessary Conditions for the Problem of Bolza, 858.

Blumenthal, L. M. Note on an Application of Metric Geometry to Determinants, 283.

Bol, G. On $n$-Webs of Curves in a Plane, 855.

Brahana, H. R. See Reviews, under Milne-Thomson.

Bridgman, P. W. Statistical Mechanics and the Second Law of Thermodynamics, 225.

Brouwer, D. See Reviews, under Moulton.

Brown, B. H. See Reviews, under Schwerdt.

Bush, L. E. Note on the Discriminant Matrix of an Algebra, 49.

Campbell, A. D. Apolarity in the Galois Fields of Order $2^{n}, 52$.

Carlitz, L. Note on Diophantine Automorphisms, 246.

- New Diophantine Automorphisms, 271.

- On Polynomials in a Galois Field, 736.

Carmichael, R. D. Note on Triple Systems, 695.

See Reviews, under Burke, Davis, Gent, Latzin, Northrop, Planck, Smart.

Carpenter, A. F. A Triad of Ruled Surfaces Defined by Reciprocal Polars, 95. 
Cauer, W. The Poisson Integral for Functions with Positive Real Part, 713. Charpentier, M. On Certain Dynamical Systems with Points of Peano, 849. Chittenden, E. W. See Reviews, under Phillips.

Clarkson, J. A. A Sufficient Condition for the Existence of a Double Limit, 391.

Clarkson, J. M. Some Involutorial Line Transformations Interpreted as Points of $V_{2}$ of $S_{5}, 533$.

Craig, C. C. See Reviews, under Camp.

Cummings, L. D. Hexagonal Systems of Seven Lines in a Plane, 105. Heptagonal Systems of Eight Lines in a Plane, 700.

Dadourian, H. M. See Reviews, under Loeb, Painlevé, Poor.

Debely, N. See Bernstern, B. A.

Decker, F. F. The Syracuse Meeting of Section A, American Association for the Advancement of Science, 601.

Dederick, L. S. See Reviews, under Sugot.

Dines, L. L. See Reviews, under Shorter.

Dodd, E. L. Frequency Laws Showing Stability with Reference to the Geometric Mean and other Means, 398.

- See Reviews, under Deltheil, Rosmanith.

Dorroh, J. L. Concerning Adjunctions to Algebras, 85.

Dresden, A. See Reviews, under University of Chicago.

Емсн, A. See Reviews, under Bieberbach, Cartan, Haussner, Müller, Schilling.

Evans, G. C. Note on the Gradient of the Green's Function, 879.

FITE, W. B. Periodic Solutions of Linear Differential Equations, 865.

Ford, L. R. See Reviews, under Bieberbach, Knopp.

ForT, T. Reports of Meetings of the American Mathematical Society: ThirtyEighth Annual Meeting, 145; March Meeting in New York, 313.

Franklin, P. See Reviews, under Hobson.

Garabedian, H. L. Note on a Theorem due to Bromwich, 541.

Gronwall, T. H. See Hille, E.

Gurney, M. Cesàro Summability of Double Series, 825.

Harry, C. H. An Examination of Some Cut Sets of Space, 427.

Hazlett, O. C. See Reviews, under Perron.

Henle, P. The Independence of the Postulates of Logic, 409.

Hildebrandt, T. H. On the Moment Problem for a Finite Interval, 269.

HILLE, E. Summation of Fourier Series, 505.

— Thomas Hakon Gronwall-In Memoriam, 775.

Hollcroft, T. R. See Reviews, under National Research Council.

Horsfall, I. O. Transformations Associated with the Lines of a Cubic, Quadratic, or Linear Complex, 872.

Hubbs, H. N. Analytic Study of Rational Quintic Surfaces having no Multiple Curves, 295.

Ingraham, M. H. Note on the Reducibility of Algebras without a Finite Base, 100.

- Reports of Meetings of the American Mathematical Society: Thanksgiving Meeting in Columbus, 1; April Meeting in Chicago, 318; ThirtyEighth Summer Meeting, 761. 
See Reviews, under Mathewson.

Karpinski, L. C. See Reviews, under Smith, Tannery.

Kennison, L. S. Reflections in Function Space, 131.

Ketchum, P. W., and Martin, T. Polygenic Functions of Hypercomplex Variables, 66.

KImbaLl, B. F. Three Theorems Applicable to Vibration Theory, 718.

Knebelman, M. S. See Reviews, under Rademacher.

Lamond, J. K. See Reviews, under Campbell.

Lane, E. P. See Reviews, under Sperry.

LANGer, R. E. See Reviews, under Volterra.

Lawton, W. On the Zeros of Certain Polynomials related to Jacobi and Laguerre Polynomials, 442.

Lehmer, D. H. Note on Mersenne Numbers, 383. An Inversive Algorithm, 693.

A Note on Fermat's Last Theorem, 723. On Euler's Totient Function, 745.

LehMer, D. N. A Correction in the List of Primes, 902.

Lindsay, R. B. See Reviews, under de Broglie.

Longley, W. R. See Reviews, under Brand, British Association for the Advancement of Science, Cahen, Dadourian, Falckenberg, Fueter, Hoheisel, Knopp, Lainé, Low, Milne-Thomson, Scarborough.

McClenon, R. B. See Reviews, under Loria.

McEwen, W. H. On the Approximate Solution of Linear Differential Equations with Boundary Conditions, 887.

MARDEn, M. A Generalization of Weierstrass' and Fekete's Mean-Value Theorems, 434.

Margenau, H. See Reviews, under Horn, von Mises.

MARIA, A. J. Examples of Harmonic Functions, 839.

Martin, T. See Ketchum, P. W.

Menge, W. O. On the Rank of the Product of Certain Square Matrices, 88.

Miller, E. W. Solution of the Zarankiewicz Problem, 831.

Miller, G. A. See Reviews, under Enciclopedia.

Mitchell, A. K. On a Matrix Differential Operator, 251.

Mitchell, U. G. See Reviews, under Archibald.

Moore, C. L. E. See Struik, D. J.

Moore, C. N. See Reviews, under Fort.

Mordell, L. J. Note on the Diophantine Equation $a x^{2}+b y^{2}+c z^{2}+d t^{2}=0,277$.

Murnaghan, F. D. See Reviews, under Boll, de Broglie, Camichel, Conservatoire, Curie, Dirac, Maxwell, Rainich.

Myers, S. B. Adjoint Systems in the Problem of Mayer under General EndConditions, 303.

Oldenburger, R. On Canonical Binary Trilinear Forms, 385.

Ore, O. See Reviews, under Kronecker, Steinitz, van der Waerden.

PALL, G. A Class of Universal Functions, 56.

Paradiso, L. J. Solutions of Bounded Variation of the Fredholm-Stieltjes Integral Equation, 255.

Parker, W. V. On Real Symmetric Determinants whose Principal Diagonal Elements are Zero, 259. 
On the Kummer Surface, 403.

A Theorem on Symmetric Determinants, 545.

Addition Formulas for Hyperelliptic Functions, 895.

Pennell, W. O. The Use of Fractional Integration and Differentiation for Obtaining Certain Expansions in Terms of Bessel Functions or of Sines and Cosines, 115.

Poor, V. C. See Reviews, under Webster.

Putnam, T. M. Report of the November Meeting of the American Mathematical Society in Pasadena, 4.

RICcI, G. On a Generalization of the Wilson-Glaisher Theorem, 393.

Richardson, R. G. D. International Congress of Mathematicians, Zurich, $1932,769$.

RIETz, H. L. On the Lexis Theory and the Analysis of Variance, 731. See Reviews, under Manes.

Ritt, J. F. See Reviews, under Appell, Dienes.

Roberts, J. H. A Property Related to Completeness, 835.

Robertson, H. P. See Reviews, under Brill.

Roth, W. E. On Algebraic Equations having Only Real Roots, 594.

Rupp, C. A. See Reviews, under Clebsch.

SAKs, S. Note on Defining Properties of Harmonic Functions, 380.

Scarborough, J. B. See Reviews, under Kryloff.

Schoenberg, I. J. On Finite and Infinite Completely Monotonic Sequences, 72. - See BLIss, G. A.

SHook, C. A. An Extension of Lagrange's Equations, 135.

- See Reviews, under Humphrey, Porter.

Sisam, C. H. See Reviews, under Haarbleicher.

Smith, D. E. See Reviews, under Brunet, Loria.

Smith, P. A. See Reviews, under von Helmholtz.

SNyder, V. See Reviews, under Coolidge, Mukhopadhyaya.

Stouffer, E. B. On the Contact of Two Space Curves, 415.

Struik, D. J. The Scientific Work of C. L. E. Moore, 155.

- See Reviews, under Duschek, Julia.

Tamarkin, J. D. On the Compactness of the Space $L_{p}, 79$.

- See Reviews, under Bieberbach, Courant, Lichtenstein, Montel, Schreier.

Thomas, T. Y. See Reviews, under McConnell.

Uspensky, J. V. See Reviews, under Dickson.

VANDiver, H. S. See Reviews, under Noguès.

Wall, H. S. On the Relationship among the Diagonal Files of a Padé Table, 752.

Walsh, J. L. On Polynomial Interpolation to Analytic Functions with Singularities, 289.

White, H. S. The Plane Figure of Seven Real Lines, 59.

WhitNey, H. A Logical Expansion in Mathematics, 572.

Whittemore, J. K. See Reviews, under Kommerell, V.

WhyBurn, W. M. On the Integration of Unbounded Functions, 123.

Wilder, C. E. See Beetle, R. D.

Wilder, R. L. A Plane, Arcwise Connected and Connected im Kleinen Point Set which is not Strongly Connected im Kleinen, 531. 
- Point Sets in Three and Higher Dimensions and their Investigation by means of a Unified Analysis Situs, 649.

Williams, A. R. The Transformation of Lines of Space by means of Two Quadratic Reguli, 554.

Wilson, W. A. On Rectifiability in Metric Spaces, 419.

On Angles in Certain Metric Spaces, 580.

Wong, B. C. On Surfaces in Space of $r$ Dimensions, 77.

- On Certain Characteristics of $k$-Dimensional Varieties in $r$-Space, 725.

Young, J. W. See BeEtle, R. D. 


\section{REviEwS}

Appell, P., et Goursat, E. Théorie des Fonctions Algébriques (deuxième édition), tome 2: Fonctions Automorphes, par P. Fatou, J. F. Ritr, 14.

Archibald, R. C. Outline of the History of Mathematics, U. G. Mitchell, 625. Artin, E. See van der Waerden, B. L.

Baer, R. See Steinitz, E.

Berzolari, L. See Enciclopedia.

Bieberbach, L. Lehrbuch der Funktionentheorie (dritte Auflage), erster Band, L. R. Ford, 19; (zweite Auflage), zweiter Band, J. D. TAMARkin, 790.

- Projektive Geometrie, A. ЕмсH, 167.

Boll, M. Exposé Electronique des Lois de l'Electricité, F. D. Murnaghan, 793. Boyce, M. G. See University of Chicago.

Braithwaite, R. B. See Ramsey, F. P.

Brand, L. Vectorial Mechanics, W. R. LongLEy, 794.

Brill, A. Vorlesungen über Allgemeine Mechanik, H. P. Robertson, 17.

British Association for the Advancement of Science. Mathematical Tables, volume $1, W$. R. LONGLey, 458.

de Broglie, L. Introduction à l'Etude de la Mécanique Ondulatoire, R. B. LiNDSAY, 166.

— Recueil d'Exposés sur les Ondes et Corpuscules, R. B. Lindsay, 166.

- Sur une Forme Plus Restrictive des Relations d'Incertitude, F. D. Murnaghan, 789.

Brunet, P. L'Introduction des Théories de Newton en France au XVIIIe Siècle. Avant 1738, D. E. Smith, 161.

Bryan, C. L. See von Helmholtz, H.

Burke, J. B. The Emergence of Life, R. D. Carmichael, 472.

Cahen, E., et Michel, C. Leçons Elémentaires sur le Calcul Numérique, W. R. LONGLEY, 335.

Camichel, C. Leçons sur les Conduites, F. D. Murnaghan, 13.

Camp, B. H. The Mathematical Part of Elementary Statistics, C. C. CraIG, 332.

Campbell, G. A., and Foster, R. M. Fourier Integrals for Practical Applications, J. K. LAMOND, 477.

Cartan, E. Leçons de Géométrie Projective Complexe, A. Емсн, 457.

Clebsch, A., und Lindemann, F. Vorlesungen über Geometrie, mit besonderer Benutzung der Vorträge von A. Clebsch bearbeitet und herausgegeben von $\mathrm{F}$. Lindemann (zweite vermehrte Auflage), erster Band, erster Teil, dritte Lieferung, C. A. RupP, 791.

Coble, A. B. See National Research Council.

Comrie, L. J. See Milne-Thompson, L. M.

Conservatoire National des Arts et Métiers. Conférences d'Actualités Scientifiques et Industrielles, année 1929, F. D. Murnaghan, 14.

Coolidge, J. L. A Treatise on Algebraic Plane Curves, V. Snyder, 163.

Courant, R., und Hilbert, D. Methoden der Mathematischen Physik (zweite verbesserte Auflage), erster Band, J. D. TAMArkin, 21. 
Curie, I., et Joliot, F. La Projection de Noyaux Atomiques par un Rayonnement très Pénétrant. L'Existence du Neutron, F. D. Murnaghan, 789.

Dadourian, H. M. Analytical Mechanics (third edition), W. R. LoNGLEY, 789.

Davis, H. T. Philosophy and Modern Science, R. D. Carmichael, 476.

- See von Helmholtz, H.

Dedekind, R. Gesammelte Mathematische Werke, herausgegeben von R. Fricke, E. Noether, O. Ore, zweiter Band, E. T. BeLL, 16.

Deltheil, R. Erreurs et Moindres Carrés, E. L. Dodd, 20.

Dickson, L. E. Studies in the Theory of Numbers, J. V. UsPensky, 463.

Dienes, P. The Taylor Series, J. F. RItr, 792.

Diès, A. See Tannery, P.

Dirac, P. A. M. Les Principes de la Mécanique Quantique, traduit par A. Proca et J. Ullmo, F. D. Murnaghan, 795.

Duren, W. L. See University of Chicago.

Duschek, A., und Mayer, W. Lehrbuch der Differentialgeometrie, erster und zweiter Band, D. J. Struik, 324.

Einstein, A. See Maxwell, J. C.

Emch, A. See National Research Council.

Enciclopedia delle Matematiche Elementari, a cura di L. Berzolari, G. Vivanti, e D. Gigli, volume 1, parte 1 e parte 2, G. A. Miller, 157.

Enriques, F. See Tannery, $P$.

Falckenberg, H. Komplexe Reihen, W. R. Longley, 330.

Fantappiè, L. See Volterra, V.

Fatou, P. See Appell, P.

Fleming, A. See Maxwell, J. C.

Fort, T. Infinite Series, C. N. Moore, 170.

Foster, R. M. See Campbell, G. A.

Fricke, R. See Dedekind, R.

Fueter, R. Das Mathematische Werkzeug des Chemikers, Biologen, Statistikers, und Soziologen (zweite verbesserte und vermehrte Auflage), W. R. LONGLEY, 330.

Fulco, P. Quozienti e Resti nella Divisione di Frazioni. Congruenze a Modulo Frazionario, E. T. BELL, 17.

Garnett, W. See Maxwell, J. C.

Gent, W. Die Raum-Zeit-Philosophie des 19. Jahrhunderts, R. D. CarMICHAEL, 330.

Gigli, D. See Enciclopedia.

Glazebrook, R. T. See Maxwell, J. C.

Goursat, E. See Appell, P.

Haack, W. See Haussner, R.

Haarbleicher, A. De l'Emploi des Droites Isotropes comme Axes de Coordonnées. Nouvelle Géométrie du Triangle, C. H. Sisam, 474.

Hasse, H. See Steinitz, E.

Haussner, R., und Haack, W. Darstellende Geometrie, dritter Teil, A. EмcH, 469.

von Helmholtz, H. Counting and Measuring, translated by C. L. Bryan with an introduction and notes by H. T. Davis, P. A. SMITH, 13 .

Hensel, K. See Kronecker, L. 
Hilbert, D. See Courant, R.

Hobson, E. W. The Theory of Spherical and Ellipsoidal Harmonics, P. FrankLIN, 479.

Hoheisel, G. Gewöhnliche Differentialgleichungen (zweite, verbesserte Auflage), W. R. LONGLEY, 478.

Horn, J. Partielle Differentialgleichungen, H. Margenau, 479.

Huke, A. See University of Chicago.

Humphrey, D. Intermediate Mechanics; Statics and Hydrostatics, C. A. Sноок, 475.

Jeans, J. See Maxwell, J. C.

Johnston, W. H. See Planck, M.

Joliot, F. See Curie, I.

Julia, G. Eléments de Géométrie Infinitésimale, D. J. Struik, 324.

Knopp, K. Funktionentheorie (vierte Auflage), erster Teil, L. R. Ford, 167; (vierte Auflage), zweiter Teil, W. R. LoNGLEy, 471.

- Aufgabensammlung zur Funktionentheorie (zweite Auflage), erster Teil, W. R. LONGLEY, 471.

Kommerell, K. See Kommerell, V.

Kommerell, V., und Kommerell, K. Theorie der Raumkurven und Krummen Flächen (vierte Auflage), erster und zweiter Band, J. K. Whitтемore, 334.

Krames, J. L. See Müller, E.

Kronecker, L. Werke, dritter Band, zweiter Teil, herausgegeben von $\mathrm{K}$. Hensel, O. ORE, 171.

Kryloff, N. Les Méthodes de Solution Approchée des Problèmes de la Physique Mathématique, J. B. Scarborovgh, 613.

Lainé, E. Exercices de Calcul Différentiel et Intégral, W. R. LongLey, 793.

Lamb, H. See Maxwell, J. C.

Lampariello, G. See Levi-Civita, T.

Larmor, J. See Maxwell, J. C.

Latzin, H. Analysis des Unendlichen im Psychischen und Physischen, R. D. CARMichael, 475.

Lefschetz, S. See National Research Council.

Levi-Civita, T. Caratteristiche dei Sistemi Differenziali e Propagazione Ondosa, lezioni raccolte da G. Lampariello, H. Bateman, 335.

Lichtenstein, L. Vorlesungen über einige Klassen nichtlinearer Integralgleichungen und Integrodifferentialgleichungen nebst Anwendungen, J. D. TAMARKIN, 625.

Lindemann, F. See Clebsch, A.

Lodge, O. See Maxwell, J. C.

Loeb, L. B. Fundamentals of Electricity and Magnetism, H. M. Dadourian, 466.

Long, M. See Volterra, V.

Loria, G. Il Passato e il Presente delle Principali Teorie Geometriche, Storia e Bibliografia (quarta edizione totalmente rifatta), D. E. SмIтн, 159.

Storia delle Matematiche, volume 2, R. B. McClenon, 787.

Low, B. B. Mathematics, W. R. LongLey, 471.

McConnell, A. J. Application of the Absolute Differential Calculus, T. Y. Thomas, 615. 
McShane, E. J. See University of Chicago.

Manes, A. Versicherungswesen, System der Versicherungswirtschaft, H. L. RIETZ, 16.

Mathewson, L. C. Elementary Theory of Finite Groups, M. H. Ingraham, 172.

Maxwell, J. C. A Commemoration Volume, 1831-1931, Essays by J. J. Thomson, M. Planck, A. Einstein, J. Larmor, J. Jeans, W. Garnett, A. Fleming, O. Lodge, R. T. Glazebrook, H. Lamb, F. D. Murnaghan, 479.

Mayer, W. See Duschek, A.

Michel, C. See Cahen, E.

Milne-Thomson, L. M. Die Elliptischen Funktionen von Jacobi, H. R. Brahana, 789.

Milne-Thomson, L. M., and Comrie, L. J. Standard Four-Figure Mathematical Tables, W. R. LoNGLey, 458.

von Mises, R. Wahrscheinlichkeitsrechnung und ihre Anwendung in der Statistik und Theoretischen Physik, H. Margenau, 169.

Montel, P. Leçons sur les Fonctions Entières ou Méromorphes, J. D. TAMARKIN, 622.

Moore, G. E. See Ramsey, F. P.

Moulton, F. R. Astronomy, D. Brouwer, 473.

Müller, E. Vorlesungen über Darstellende Geometrie, dritter Band: Konstruktive Behandlung der Regelflächen, bearbeitet von J. L. Krames, A. Емсн, 476.

Mukhopadhyaya, S. Collected Geometrical Papers, part 2, V. Snyder, 480.

National Research Council. Bulletin of the National Research Council, No. 63: Selected Topics in Algebraic Geometry, by V. Snyder, A. B. Coble, A. Emch, S. Lefschetz, F. R. Sharpe, C. H. Sisam, T. R. Hollcroft, 7. Noether, E. See Dedekind, R., van der Waerden, B. L.

Noguès, R. Théorème de Fermat, son Histoire, H. S. VAndrver, 794.

Northrop, F. S. C. Science and First Principles, R. D. Carmichael, 472.

Ore, O. See Dedekind, R.

Painlevé, P. Cours de Mécanique, tome 1, H. M. Dadourian, 466.

Perron, O. Algebra (zweite, verbesserte Auflage), erster Band, O. C. Hazlett, 623.

Phillips, E. G. A Course of Analysis, E. W. Chittenden, 168.

Planck, M. The Universe in the Light of Modern Physics, translated by W. H. Johnston, R. D. CARMICHAEL, 173.

- See Maxwell, J. C.

Poor, V. C. Electricity and Magnetism, H. M. Dadourian, 12.

Porter, A. W. Thermodynamics, C. A. Sноoк, 479.

Proca, A. See Dirac, P. A. M.

Rademacher, H., und Toeplitz, O. Von Zahlen und Figuren, M. S. KnebelMAN, 15.

Rainich, G. Y. Mathematics of Relativity, F. D. Murnaghan, 790.

Ramsey, F. P. The Foundations of Mathematics, and other Logical Essays, edited by R. B. Braithwaite, preface by G. E. Moore, B. A. Bernstein, 611.

Rosmanith, G. Mathematische Statistik der Personenversicherung, E. L. DoDd, 18. 
Scarborough, J. B. Numerical Mathematical Analysis, W. R. Longley, 331. Schilling, F. Die Pseudosphäre und die Nichteuklidische Geometrie, A. Емсн, 335.

Schreier, O., und Sperner, E. Einführung in die Analytische Geometrie und Algebra, erster Band, J. D. TAMARKIN, 622.

Schwerdt, H. Die Anwendung der Nomographie in der Mathematik, B. H. BROWN, 16.

Sharpe, F. R. See National Research Council.

Shorter, L. R. Introduction to Vector Analysis, L. L. Dines, 468.

Sisam, C. H. See National Research Council.

Smart, H. R. The Logic of Science, R. D. Carmichael, 475.

Smith, D. E., edited by. A Source Book in Mathematics, L. C. KARPINSKI, 333.

Snyder, V. See National Research Council.

Sperner, E. See Schreier, O.

Sperry, P. Bibliography of Projective Differential Geometry, E. P. Lane, 19.

Steinitz, E. Algebraische Theorie der Körper, herausgegeben von R. Baer und H. Hasse, O. Ore, 22.

Sugot, G. Balistique Intérieure Théorique et Tables Numériques, L. S. DEDERICK, 467.

Szegö, G. See Webster, A. G.

Tannery, P. Pour l'Histoire de la Science Hellène. De Thalès à Empédocle (deuxième édition par A. Diès, avec une préface de F. Enriques), L. C. KARPINSKI, 470.

Thomson, J. J. See Maxwell, J. C.

Toeplitz, O. See Rademacher, H.

Ullmo, J. See Dirac, P. A. M.

University of Chicago. Contributions to the Calculus of Variations, 1930. Theses submitted to the Department of Mathematics of the University of Chicago, by M. G. Boyce, A. Huke, F. L. Wren, E. J. McShane, W. L. Duren, A. DRESDEN, 617.

Vivanti, G. See Enciclopedia.

Volterra, V. Theory of Functionals and of Integral and Integro-Differential Equations, edited by L. Fantappiè, authorized translation by M. Long, R. E. LANGER, 623.

van der Waerden, B. L. Moderne Algebra, unter Benutzung von Vorlesungen von E. Artin und E. Noether, erster und zweiter Band, O. ORE, 327.

Webster, A. G., und Szegö, G. Partielle Differentialgleichungen der Mathematischen Physik, V. C. Poor, 15.

Wren, F. L. See University of Chicago.

Abstracts of Papers Presented to the Society, 27, 177, 343, 486, 632, 800.

Errata, 194, 456, 935.

Index of Volume XXXVIII, 919.

New Publications, 43, 195, 355, 497, 643, 820.

Notes, 24, 174, 336, 481, 627, 796.

Papers Read before the Society and Subsequently Published, Forty-First Annual List of, 903. 


\section{NOTES AND OTHER ITEMS}

Academies, Associations, Congresses, and Societies:

American Mathematical Society: Auditors' Report, 148; Budget, 149; Bulletin, 796; Cole Prize, 146; Colloquium Publications, 761, 796; Committee on Annual Meeting, 149; on Gibbs Lecture, 149; on Nominations, 149; on Semi-Centennial, 149; Dates and Places of Meetings, 4, 149, 319, 764; Delegates to International Mathematical Congress, 627, 770; Election of Officers, 148; Endowment Fund, 148; Gibbs Lecture, 145, 149, 150; Librarian's Report, 149; New Members Admitted, 146, 147, 148, 319, 762, 763; Representatives on Council of the American Association for the Advancement of Science, 149; on Editorial Board of the American Year Book, 763; on National Research Council, 149; Statistics, 148; Transactions, 174, 796; Treasurer's Report, 148.

Associations for the Advancement of Science: American, 149, 174, 336, 601; British, 25, 481.

Accademia dei Lincei, 25; Acoustical Society of America, 24; American Institute of Physics, 24, 627; American Physical Society, 24; Amsterdam Mathematical Society, 481; Astrophysical Union, 796; Belgian Academy, 336; British Institution of Electrical Engineers, 336; Comité International d'Histoire des Sciences, 336; Franklin Institute, 481; Indian Mathematical Society, 24; International Committee on the Teaching of Mathematics, 772; International Mathematical Congress, 24, 174, 627, 769, 770, 773; International Mathematical Union, 773; Kieff Academy of Sciences, 336; London Mathematical Society, 24; Mathematical Association of America, 336, 770; National Research Council, 149, 628; Optical Society of America, 24, 25; Paris Academy of Sciences, 174; Prussian Academy of Sciences, 481; Roosevelt Memorial Association, 481; Royal Astronomical Society, 336; Royal Society of London, 25; Society of Rheology, 24; Unione Matematica Italiana, 481.

Books, Announcement of New, 796, 796.

Doctorates in Mathematics, American, 338, 629.

Fellowships: Guggenheim, 337; National Research, 628; Sterling, 482.

Journals:

American Mathematical Monthly, 24; American Year Book, 763; Bulletin of the American Mathematical Society, 796; Ergebnisse eines Mathematischen Kolloquiums, 481; Jahrbuch über die Fortschritte der Mathematik, 481; Journal of Chemical Physics, 627; Journal of Science of the Hiroshima University, 481; Kieff Academy of Sciences, Bulletin de la Classe des Sciences Naturelles et Techniques, 336, Journal du Cycle Mathématique, 336; Popular Science Monthly, 336; Revue d'Acoustique, 627; Revue Semestrielle des Publications Mathématiques, 481; Schriften des Mathematischen Seminars und des Instituts für Angewandte Mathematik der Universität Berlin, 627; Science Reports of the National Tsing Hua University, 336; Transactions of the American Mathematical Society, $174,796$. 
Papers and Communications Presented to the Society, Authors:*

Adkisson, V. W., 3 (37; 833); Agnew, R. P., 154 (41), 318 (344); Aitchison, B., 769 (800); Albert, A. A., 152 (33), 322 (180), 322 (179), 323 (179), 766 (488), 766 (496); Albert, O. W., 6 (37; 825); Allen, E. S., (813); Archibald, R. G., 152 (34); Atanasoff, J. V., (816); Ayres, W. L., 2 (27).

Bacon, H. M., 765 (634); Ballantine, J. P., 764 (490); Bamforth, F. R., 153 (38); Barnard, R. W., 322 (350); Barnett, I. A., 320 (345); Bartky, W., 322 (350), 768 (492); Basoco, M. A., 3 (37; 832), 321 (349); Baten, W. D., 2 (37; 827), 321 (181); Bell, C., 767 (353), 768 (352); Bell, E. T., 5 (37; 822), 764 (494); Bernstein, B. A., 764 (802), 768 (637); Binney, J. H., 766 (801); Birkhoff, G. D., 145, 322 (350); Black, A. H., 768 (490); Blake, A., (805); Bliss, G. A., 145, 321 (347), (640); Blumenthal, L. M., 152 (35), 315 (182); Botsford, J. L., 5 (37; 823), 322 (351), 767 (640), 767 (801); Bourgin, D. G., 153 (41); Bower, O. K., 765 (486), 765 (486); Brahana, H. R., 767 (488), 767 (488); Brandner, F. A., (813); Brenke, W. C., (816); Bridgman, P. W., 145; Brown, A. B., 314 (183); Brown, B. H., 317 (192); Bruton, G. S., 766 (633); Burington, R. S., 322 (180); Bush, L. E., $2(37 ; 830), 2(37 ; 830)$.

Cairns, S. S., 317 (192), (806), (807); Cameron, R. H., (817); Camp, B. H., (804); Camp, C. C., 321 (181); Campbell, A. D., 152 (33), (802), (819); Carlitz, L., 315 (185), 315 (185), 315 (186), 767 (488), 767 (489), 767 (489); Carmichael, R. D., 146, 765 (489), 766 (354), 768 (492); Cauer, W., 766 (640); Chittenden, E. W., 3 (37; 832); Church, A., 768 (486); Churchill, R. V., 3 (37; 830); Clarkson, J. M., 317 (193); Clifford, A. H., 5 (37; 823), 767 (640); Cohen, L. W., 3 (37; 833), 154 (40); Coleman, J. B., 150 (28); Comenetz, G., 317 (192), (803); Conwell, H. H., 322 (37; 834); Coral, M., 766 (637); Courant, R., 314; Court, N. A., 151 (32), 767 (493); Craig, A. T., 150 (28), 150 (29), 321 (346), (813); Craig, H. V., 316 (188); Cummings, L. D., 317 (193); Currier, A. E., 323 (352), (489); Curry, H. B., 153 (37), 768 (353).

Davis, H. T., 2 (37; 828), 154 (39), (814); Deutsch, J. G., 765 (487), (806), (809), (809); Dix, C. H., 152 (35), 153 (37); Dodd, E. L., 150 (29); Doob, J. L., 316 (188), 317 (190); Dorroh, J. L., 315 (185); Dressel, F. G., 315 (343), 317 (344); Durfee, W. H., 321 (348); Dushnik, B., 2 (37; 827); Dye, L. A., 151 (31).

Edmonson, N., 154 (40); Emch, A., 3 (37; 831); Evans, G. C., 766 (801).

Fialkow, A., (803); Fischer, C. H., 150 (28); Ford, L. R., 151 (32); Foster, A. L., 767 (491); Foster, M. C., 316 (193); Foster, R. M., 3 (37; 833); Frink, O., (641); Fuller, G., 320 (345).

Garabedian, H. L., 765 (354); Garver, R., 5 (37; 823), 315 (186), 766 (638), 768 (638), 769 (638); Gehman, H. M., 1; Gergen, J. J., 316 (189), (809); Germond, H. H., 150 (27), 150 (29); Glenn, O. E., 322 (351); Goldberg, M., 151 (37; 834); Grant, J. D., 765 (486); Graustein, W. C., 1; Gray, M. C., (815); Groat, B. F., 768 (353).

Hadlock, E. H., (805); Hall, M., 315 (184); Hasse, H., 322 (179); Hedrick, E. R., 145, 765 (492); Hestenes, M. R., 321 (347), 321 (348);

* Numbers within parentheses refer to abstracts, and those outside parentheses to titles as listed in reports of meetings. 
Hildebrandt, T. H., 765 (634); Hille, E., 314; Hodge, W. V. D., 317 (190); Holl, D. L., (815); Hopf, E., 152 (35); Horsfall, I. O., (802); Hotelling, H., (812); Hull, R., 767 (491); Huntington, E. V., 152 (42), 768 (493), (810); Hurwitz, W. A., 314, 318 (345).

Ingraham, M. H., 766 (633), (814).

Jackson, D., 764 (496), (816); James G., 766 (634); Jeffery, R. L., 315 (42), 323 (352); Jones, B. W., 151 (31).

Kasner, E., 316 (187), (811); Kennison, L. S., 316 (177), 317 (177); Kimball, B. F., 315 (186), 317 (187); Korzybski, A., 152 (36); Kusner, J. H., 314 (182).

Lanczos, C., 2 (37; 829); Lane, E. P., 320, 321 (180); LaPaz, L., 2 (37; 826); Latimer, C. G., 151 (31); Lawton, W. S., 317 (191); Lefschetz, S., 765 (495); Lehmer, D. H., 5 (37; 822), 5 (37; 823), 766 (635), 767 (635); Lehmer, D. N., 761; Leighton, W., (641); Lev, J., (817); Levine, A. H., 5 (37; 825); Littauer, S. B., (808), (809); Lorch, E. R., 317 (42); Lovell, C. A., 768 (487); Luther, C. F., 766 (632).

McCormick, E. O., 768 (488); McCoy, N. H., (818); McEwen, W. H., 764 (492); McGiffert, J., 765 (641); McShane, E. J., 153 (37; 523), (810), (810); MacColl, L. A., (807); MacMillan, W. D., 768 (492); Manning, W. A., 322 (350), 764 (634); Marden, M., 154 (39); Martin, R. S., 5 (37; $824), 5(37 ; 824), 5(37 ; 824), 5(37 ; 824), 5(37 ; 824), 321$ (349), 765 (639), 765 (801); Matison, H., 5 (37; 825), 321 (348); Michal, A. D., 5 (37; 825), 322 (351), 765 (639), 765 (801), 767 (640), 767 (801); Miles, E. R. C., 317 (344); Miller, E. W., (642); Miller, H. L., 766 (633); Miller, P. C., 3 (37; 831); Mitchell, A. K., 317 (187), (803); Moore, C. N., 2 (37; 827), 316 (186); Moore, R. L., 4; Morrey, C. B., (809); Morse, M., (808); Moskovitz, D., (815); Myers, S. B., 317 (191).

Nathan, D. S., 321 (346).

Oakley, C. O., 151 (32); Odoms, A., 766 (637); Oldenburger, R., 323 (181); Ore, O., 315 (184).

Pall, G., 6 (27), 152 (33), 152 (34), (808); Perlo, V., (804); Pollock, S., 2 (37; 828); Prenowitz, W., 318 (345); Purcell, E. J., 767 (636); Putnam, R. G., 153 (37); Pyle, H. R., (814).

Radó, T., 2 (37; 828), 320 (346), 322 (351), 761; Rainich, G. Y., 2 (37; 829); Raudenbush, H. W., 765 (495); Rietz, H. L., 150 (28); Ritt, J. F., 761; Roberts, J. H., 314 (177); Robertson, F., (817); Robertson, H. P., 768 (800); Robinson, S., 3 (37; 832), 321 (347), (807); Rosinger, K. E., (805); Ross, A. E., 766 (636), 767 (632); Roth, W. E., 2 (37; 830); Russell, H. G., (490); Rust, W. M., (642); Rutledge, G., 316 (189), 316 (189).

Saks, S., (812); Schoenberg, I. J., 3 (37; 832), 154 (40), 322 (349), 765 (634), 765 (639), (640); Schoonmaker, H. E., 151 (37; 834); Schottenfels, I. M., 321 (347); Seidel, W., (806); Sharpe, F. R., 151 (31); Shaub, H. C., 317 (33); Shaw, A. A., 6 (37; 826), 768 (638); Sheffer, I. M., 152 (34); Sherman, J., 317 (191); Shover, C. G., 2 (37; 829); Simmons, H. A., 151 (42), 323 (351); Singer, J., (495); Sinkov, A., 315 (184); Slotnick, M. M., 151 (32); Smith, P. F., 316 (194); Snyder, V., 151 (37; 834), (812); Sperner, E., 768 (800); Stephens, R. C., 152 (36), 768 (633); Stouffer, E. B., 322 (179), 322 (180); Swann, W. F. G., 145; Synge, J. L., 152 (35), 153 (36). 
Thielman, H. P., 3 (27); Thomas, J. M., 3 (37; 831), 154 (39), 316 (187); Trjitzinsky, W. J., 322 (350), (806), (811); Turner, J. S., 322 (350).

Wadsworth, G. P., 765 (494); Wall, H. S., 321 (181), 766 (639); Walsh, J. L., 316 (178), 316 (178), 316 (178), 316 (178), 316 (178), (490), (811), (818); Ward, M., 766 (635), 766 (636), 766 (635); Wells, C. P., (817); Wertheimer, A., 151 (30); Wexler, C., 767 (641); Whitehead, J. H. C., 765 (495); Whitney, H., 153 (37), 153 (37), 153 (38), 153 (38), 154 (41), 315 (183), 315 (183), 768 (354), (808); Whyburn, G. T., $153(37 ; 834), 315$ (179), 764 (639), (818), (819); Whyburn, W. M., 5 (37; 822), 765 (494), 765 (492); Wiener, N., (642); Wilder, R. L., 2 (37; 828), 320; Wilks, S. S., 150 (29), 151 (30), 316 (190), 317 (188); Williams, A. R., 6 (37; 826); Wilson, W. A., 315 (184), 315 (184), (804), (811); Wilson, W. H., 154 (38);

Wong, B. C., $6(37 ; 826)$; Worth, C. R., $322(350)$.

Young, R. C., (642).

Zariski, O., 315 (343); Zippin, L., 314 (343), 314 (182), (803); Zygmund, A., 316 (344).

\section{Personal Notes:}

Abetti, G., 25; Adams, C. R., 148, 482; Adams, P. A., 484; Aitken, R. G., 336; Albert, O. W., 629; Alexander, J. W., 770; Alliaume, M., 176; Ames, D. B., 338; Appell, P., 174; Archibald, R. C., 770; Armstrong, B. M., 483; Ayres, W. L., 796.

Babbage, D. W., 336; Backhaus, H., 628; Bailey, H. W., 483; Baire, R., 631; Baldus, R., 337; Bamberger, L., 797; Barbarin, P., 484; Barnard, R. W., 630; Barton, H., 628; Barton, H. A., 24; Bauer, L. A., 485; Beale, F. S., 338; Beckenbach, E. F., 628; Beghin, H., 482; Bennett, A. A., 482; Benton, T. C., 484; Bickley, W. G., 175; Bieberbach, L., 627; Bigourdan, G., 342; Birkhoff, G. D., 24; Blaschke, W., 175, 482; Blichfeldt, H. F., 770; Bliss, G. A., 24; Bohnenblust, H. F., 338; Borofsky, S., 338; Bouligand, G., 175; Bowie, W., 797; Bragg, W. L., 25, 481, 628; Bridgman, P. W., 481; Brillouin, J., 627; Brillouin, L., 627; Brodie, W. M., 799; de Broglie, L., 797; de Broglie, M., 627; Brown, B. H., 483; Brown, E. W., 176; Brown, O. E., 798; Buchanan, H. E., 336; Buckman, A. L., 799; Buisson, H., 627; Bullock, R. C., 798; Bumer, C. T., 26, 629; Burington, R. S., 629; Burke, L., 338; Bush, L. E., 338, 484; Bush, V., 341; Bussey, W. H., 24, 336; Butter, F. A., 484; Byrne, W. E., 483.

Cabannes, J., 627; Caccioppoli, R., 628; Cairns, S. S., 338; Cairns, W. D., 770; Calkins, H., 338; Cantone, M., 484; Cardwell, A. B., 338; Carlitz, L., 630; Carnap, R., 482; Carroll, E. T., 338; Carver, W. B., 24; Chace, A. B., 631; Chandler, E. M., 338; Chapman, S., 24; Chazy, J., 174; Chipart, H., 174; Chittenden, E. W., 148; Chrétien, H., 174; Church, W. R., 484; de la Cierva, J., 481; Coble, A. B., 148, 149, 149; Compton, A. H., 797; Coolidge, J. L., 149; Coral, M., 338; Cossar, J., 336; Courant, R., 482; Coxeter, H. S. M., 26; Craig, A. T., 338; Craig, C. C., 630; Curtiss, D. R., 174.

Dantzig, T., 342; Debye, P., 337, 627; Den Hartog, J. P., 483; Deuring, M. F., 482; Devaux, H., 174; Didot, H., 337; Dillon, J. H., 338; Dingler, H., 628; Dirac, P. A. M., 482, 629; Dixon, A. C., 24, 797; Dörge, K., 628; Donahue, J. E., 631; Donati, L., 485; Donohue, J. E., 338; Doob, 
J. L., 628; Dorroh, J. L., 483; Dorwart, H. L., 338; Douglass, R. D., 338; Downs, T. L., 484; Dresden, A., 482; Dushnik, B., 339; von Dyck, W., 481.

Earl, J. M., 483; Eastham, J. N., 339; Echols, C. P., 26; Eddington, A., 482, 796; Edge, W. L., 629; Eggers, H. C. T., 339; Einstein, A., 175, 627, 797; Emch, A., 148; Evans, G. C., 336; Ewing, J. A., 481.

Fantappiè, L., 629; Faris, R. L., 799; Farnum, F., 798; Farrell, O. J., 483; Feld, J. M., 339, 484, 631; Fields, J. C., 631, 773; Fine, H. B., 24; Finzi, B., 629; Fischer, C. H., 484; Fisk, N. C., 339; Fithian, J. H., 483; Flexner, A., 797; Flexner, W. W., 483; Flood, M. M., 484; Ford, W. B., 336; Fort, T., 763; Foster, A. L., 339; Fowler, R. H., 337; Frame, J. S., 484; Friedli, W., 25; Fubini, G., 627; Fueter, R., 770; Fuld, 797.

Galbraith, A. S., 484; Garnier, R., 174; Germay, 336; Germond, H. H., 483; Gerst, F. J., 483; Gibbs, J. W., 176; Glashan, J. C., 342; Glover, J. W., 342; Gödel, K., 481; Goldschmidt, V. M., 25; Gough, M. de L., 339; Gourin, E., 339; Graesser, R. F., 483; Graham, P. H., 798; Gronwall, T. H., 342 .

Hadamard, J. S., 627, 772; Hadley, S. M., 26; Hagen, B. L., 484; Hall, F. C., 799; Hardy, G. H., 26; Hartmann, F. M., 342; Hartree, D. R., 337; Hartung, M. L., 339; Hatcher, T. W., 339; Hedrick, E. R., 336, 337, 628; Hefner, R. A., 339, 483; Heisenberg, W., 483; Hemenway, L. D., 483; Herbrand, J., 174; Hestenes, M. R., 630; Hicks, H. C., 630; Hildebrandt, T. H., 149; Hille, E., 482; Hilton, H., 24; Hinsch, V. B., 630; Hoersch, V. A., 630; Hollcroft, T. R., 148, 175; Holmes, C. T., 339; Hopf, E., 798; Hubbs, H. N., 339; Huber, C. M., 631; Hughes, H. J., 799; Hume, A., 798; Hutcherson, W. R., 339.

Ince, E. L., 26, 629; Inglis, D. R., 339; Ingold, L., 149; Ingraham, M. H., 148, 149, 176.

Jackson, D., 628; Jackson, R. L., 630; Jacoby, H., 631; Jaeger, C. G., 483; James, G. O., 26; James, R. D., 628; Jeans, J. H., 337, 482; John, F. W., 798; Johnson, E. H., 631; Johnson, N., 799; Julia, G., 174.

Karl, M. C., 339; Kasner, E., 627; Kellogg, O. D., 631; Kennison, L. S., 484, 629; Keyser, C. J., 336; Kholodovsky, E. A., 26; Kirchner, F., 25; Kline, J. R., 149; Knebelman, M. S., 149; Knisely, A., 176; Koenigs, G., 176, 337, 482; Kokomoor, F. W., 630; Kormes, J. P., 484, 631; Kovarik, A., 797; Kryder, L., 342.

Lagarde, I., 174; Lanczos, C., 484; Lane, E. P., 174; Langmuir, I., 336, 797; Laplace, P. S., 796; Larmor, J., 629; Lefschetz, S., 149; Lepage, 336; Levy, H., 24 ; Lieber, H. G., 484; Lodge, O., 336; Longley, W. R., 148, 336; Lorentz, H. A., 175; Lucas, R., 627; Lyman, T., 25.

McCarthy, E. D., 630; McCrea, W. H., 629; McDonough, D., 339; MacDuffee, C. C., 148; Maggi, G. A., 629; Mammana, G., 337; Manning, W. A., 149; Marcelin, A., 627; Marshall, D. H., 342; Martin, M. H., 628; Martin, R. S., 628; Mayer, W., 25, 797; Meder, A. L., 798; Mendel, C. W., 628; Menge, W. O., 339, 484; Menger, K., 481; Mercer, J., 342; Merrill, J. E., 484; Miller, H. L., 339; Miller, N., 630; Millikan, R. A., 481, 628; Milne, W. E., 148, 797; von Mises, R., 627; Mitchell, H. H., 148, 174; Mohrmann, H., 25; Moore, C. L. E., 26; Moore, C. N., 337, 627, 770; 
Moore, E. H., 148; Moore, G. E., 339; Moore, R. L., 148, 149, 482; Morrey, C. B., 340, 628; Morris, R., 149; Morrison, C. M., 340; Morse, M., 174, 482, 770; Morton, A. B., 149; Moser, C., 25; Mosesson, Z. I., 484; Moskovitz, D., 630; Moston, L. T., 484; Mott, N. F., 482; Moursund, A. F., 631; Mullings, M. E., 340; Mullins, G. W., 148, 149; Murnaghan, F. D., 149; Myers, G. W., 26.

Nagel, T., 25; Narliker, V. V., 336; Newman, M. H. A., 24; Newsom, C. V., 340; Nielsen, N., 26; Nobile, V., 25; Nöbeling, G., 481; Norlie, O. M., 798; Northrop, E. P., 631; Northrop, F. S. C., 337; Norton, H. T. J., 24 ; Nyswander, J. A., 176.

Ogg, F. C., 630; Olds, E. G., 340, 630; Oort, J. H., 797; Ostenfeld, A., 485; Ostwald, W., 342; O'Toole, A. L., 340.

Painlevé, P., 481; Paradiso, L. J., 340; Parker, W. V., 340; Parodi, H., 174; Peano, G., 485; Picard, E., 25, 175, 627; Picone, M., 629; Pierce, J., 340; Pierpont, J., 149; Pixley, H. H., 340; Plancherel, M., 25; Planck, M., 796; Pollock, S., 340; Pontrjagin, L. S., 336; Post, E. L., 799; Powell, J. E., 340, 484; Price, G. B., 631; Priestley, H. J., 342; Proca, A., 627; Pupin, M. I., 25; Putnam, T. M., 148; Pyle, H. R., 340.

Raman, C. V., 796, 797; Reddick, H. W., 148; Rees, M. S., 340, 630; Rice, E. W., 175; Richardson, A. R., 337; Richardson, R. G. D., 148, 149, 627, 770; Ritt, J. F., 337; Roberts, B. D., 630; Roberts, B. J., 340 ; Robertson, H. P., 149; Robinson, H. A., 340; Robinson, L. V., 630; Robinson, S. L., 340, 628; Roman, I., 26; Rosenthal, A., 629; Ross, A. E., 340, 628; Rouse, T. A., 340; Russell, H. N., 175; Russell, J., 628; Rutherford, E., $25,25,482,482$.

Sanger, R. G., 340; Sauer, R., 797; Sbrana, F., 629; Scherberg, M. G., 340 ; Schilling, B., 175; Schmeiser, M. F., 341; Schmidt, E., 627; Schur, F., 485; Schur, I., 627; Scorza, G., 481; Scott, C. A., 26; Segre, B., 629; Seidel, W., 484; Service, J. H., 758; Shewhart, W. A., 337; Shover, C. G. 341; Shuster, A., 25; Siegbahn, M., 25; Simpson, C. G., 485; Singer, J., 341; de Sitter, W., 175, 627; Slaught, H. E., 628; Slepian, J., 627; Slobin, H. L., 798; Smith, D. E., 770, 772; Smith, P. A., 149; Smith, P. K., 341; Smith, T. L., 341; Snyder, M. B., 799; Snyder, V., 149; Sokolnikoff, I. S., 341; Sommerfeld, A., 481; Spampinato, N., 629; Sparks, F. W., 341; Steen, F. H., 484; Stephens, R. C., 341; Stone, M. H., 174, 770; Stouffer, E. B., 627, 770; Stratton, W. T., 341; Stroobant, P., 481; Struve, O., 630; Swain, G. F., 799; Swift, E., 26; Swingle, P. M., 628.

Tamarkin, J. D., 148, 627, 770; Tanzola, J. J., 148; Tate, J. T., 24; Taylor, H. M., 336; Taylor, M. E., 341; Temple, G. F. J., 175, 337; Thomas, J. M., 628; Thompson, E. L., 484; Thompson, W. R., 798; Thomson, J. F., 799; Thomson, J. J., 25; Tonelli, L., 628; Torelli, G., 176; Torrance, C. C., 341; Torrey, M. M., 798; Trjitzinsky, W. J., 484; Tucker, A. W., 628; Turnbull, H. W., 337.

Urey, H. C., 627; Urner, S. E., 631.

Vanderslice, J. L., 797; Vandiver, H. S., 146; Vaudreuil, M. F., 341; Veblen, O., 149, 176, 628, 770, 797; Villat, H., 337; Vitali, 485; Volk, O., 797; Volterra, V., 337.

Wagner, C. C., 341, 631; Warnock, W. G., 341; Watson, G. N., 24; 
Watts, C. W., 26; Weaver, W., 176; Wegner, K. W., 484; Weiss, E. A., 629; Western, A. E., 24; Weyl, H., 798; Wheeler, A. P., 628; Wheeler, H. E., 341; White, F. P., 24; White, H. S., 628; Whitehead, A. N., 482; Whitford, D. E., 176; Whitney, H., 483, 628; Whittaker, E. T., 25, 628; Widder, D. V., 482; Wieleitner, H., 176; Wiener, N., 631; Wilczewski, J., 798; Wilczynski, E. J., 799; Wilks, S. S., 341, 628; Williams, K. P., 149; Wiman, A., 25; Winch, R. P., 341; Wolf, M., 799; Wong, Y. K., 341; Wood, F., 484; Wood, R. B., 176; Worth, C. R., 631; Wren, T. L., 24.

Young, J. W., 149, 176, 320; Young, L. C., 26.

Zariski, O., 484 ; Zeigel, M. L., 798; Zippin, L., 484.

Prizes and Medals:

Accademia dei Lincei, 25; American Mathematical Society, Cole Prize, 146; Belgian Academy, de Potter Prizes, 336; British Institution of Electrical Engineers, Faraday Medal, 336; Cambridge University, Smith's, Rayleigh Prizes, 336; Comenius University, Memorial Medal, 797; Edison Medal, 175; Franklin Institute, Cresson Medal, 481; Fritz Medal, 25; Goethe Medal, 796; Guggenheim Medal, 481; International Mathematical Congress, Fields Medals, 773; Optical Society of America, Ives Medal, 25; Paris Academy, 174; Popular Science Monthly, 336; Roosevelt Memorial Association, 481; Royal Astronomical Society, 336; Royal Society of London, Sylvester, Hughes, Copley Medals, 25; John Scott Award, 627; Unione Matematica Italiana, Fubini Prize, 481; University of Michigan, Henry Russell Award, 796; University of Moscow, 336.

Universities and Technical Schools:

Berlin, 627; Cambridge, 336; Comenius, 797; Freiburg, 25; Hiroshima, 481 ; Institute of Advanced Study, 797 ; Michigan, 796; Moscow, 336; Oregon State Agricultural College, 797 ; Oregon State University, 797 ; Princeton, 24, 797; Tsing Hua, 336; Yale, 176.

\section{ERRATA}

Page 406, line 25: for $-2 x y^{3} z$ read $-2 x y^{2} z$.

Page 482, line 27: for Plaschke read Blaschke.

Page 490, line 9: for Mr. H. G. Russell read Dr H. G. Russell.

Page 546, line 22: for $(\gamma u+\delta v-\epsilon u)$ read $(\gamma w+\delta v-\epsilon u)$.

Page 629, line 36: for T. C. Bumer read C. T. Bumer. 